\title{
Evaluation of Economic Load Dispatch Problem in Power Generating Stations by the Use of Ant Colony Search Algorithms
}

\author{
M.N. Nwohu, and Osaremwinda Osarobo Paul
}

Department of Electrical/Electronics Engineering, Federal University of Technology, Minna.

\begin{abstract}
Power utilities in the world are required to ensure that electrical energy demands from the customer aremet in accordance with the policy of the country. They are also to ensure that the electrical power is generated within minimal cost when serving the load demands of the country. Thus, the total load demand must be approximately shared among the generating units with an objective to minimize the total generation cost for the system in order to satisfy the economic operation of the system. Economic load dispatch is a procedure to determine the electrical power to be generated by the committed generating units in a power system so that the total generation cost of the system is minimized, while satisfying the load demand, equality and inequality constraints simultaneously. This paper presents the economic load dispatch problem solved using Ant Colony Search Algorithm(ACSA) technique. ACSA is a meta-heuristic algorithm for solving hard combinatorial optimization problems. In this paper, the proposed algorithm was written in MATLAB code and was tested using Egbin Thermal Power Station in Nigeria as our case study with six generating units. The result obtained using ACSA was compared with the Lambda method. The results revealed that the proposed algorithm has the merit in achieving optimal solution for addressing the operating cost and power loss problems.
\end{abstract}

Keywords: Economic Load Dispatch, Ant Colony Search Algorithms, Operating Cost, Power plants, Optimization

\section{INTRODUCTION}

The effective economic operation and management of electrical power generating system has always been an important concern in the electrical power industry. The growing size of power grids, huge load demand and continuous rise in price of fossil fuel necessitate the optimal combination of generation level of power generating units. The problem of Economic Load Dispatch (ELD) is to minimize the total cost of power generation (which includes fuel consumption and operational cost) of power plants while satisfying the various loads demand and losses in the power transmission system. The objective is to distribute the total load demand and total loss among the generating plants while simultaneously minimizing generation costs and satisfying the operational constraints. The Economic Load Dispatch requires the generation facilities to plan and forecast optimal energy dispatch. Several considerations are made during energy scheduling, these amongst others are to determine the existing generating units, the distance between load centres and the generating unit, identifying the operating limits of each generating units such as the ramp rate, maximum and minimum generation level and the maximum amount of the permissible running time for the generating unit. The Economic Load Dispatch was first investigated by the use of digital computer in 1954 and is being used to date [1].

Over the past decades, there have been a lot of methods employed for solving Economic Load Dispatch. These methods amongst others include mathematical programming (classical calculusbased techniques), artificial intelligence and hybrid optimization techniques. The practical generation problems such as multi fuel, valve point effect, prohibited zone, make Economic Load Dispatch become the non-convex problems which are more difficult to solve. The non-convex optimization problem is still a challenge for conventional methods[2]. Hence, there is a need to develop new techniques for solving non-convex problems, especially for large-scale systems. Recently, modern heuristic optimization techniques have been applied to solve Economic Load Dispatch due to their abilities to find an almost global optimal solution. The search for a global optimum to an optimization problem is conducted by moving from an old population of individuals to a new population using foraging behaviour. Ant Colony Search Algorithms searches multiple solutions simultaneously in contrast to conventional optimal algorithms. This is being used in many optimization applications today [3]. 


\section{ECONOMIC LOAD DISPATCH}

For interconnected systems, the main goal of the Economic load Dispatch is to find the real and reactive power scheduling in order to reduce the cost function of different generating units in the power system. Hence, for economic operation of the system, the total load demand must be optimally shared among all the generating units with an objective to minimize the total generation cost [1]. Economic load dispatch (ELD) is a procedure to schedule the optimal combination of generation of all generating units in power system so that the total generation cost of system is minimized, while satisfying the load demand, system equality and inequality constraints. Economic load dispatch explores the best way to minimize the current generator operating costs. The essential operation constraints are the power balance constraint, that is, the total generated power being equal to the load demands plus the transmission losses on the electrical network, and the power limit constraints of the generating units. The problem of economic operation of a power system or optimal power flow (OPF) is the allocation of the load (MW) among the various units of generating station and among the various generating stations in such a way that, the overall cost of generation for the given load demand is minimum. This is an optimization problem, the objective of which is to reduce the power generation cost function subject to the satisfaction of a given set of equality and inequality constraints. For a given load demand, power flow study can be used to calculate active and reactive power generations, line flows and losses. The study also furnishes some control parameters such as the magnitude of voltage and voltage phase differences. The economic scheduling problem can be understood as an outcome of multiple power flow studies, where a particular power flow study result is considered more appropriate in terms of cost of generation. The solution to this problem cannot be optimal unless otherwise all the constraints of the system are satisfied. The problem of economic operation of the power system involves two sub-problems, namely, unit commitment (UC) and economic dispatch (ED). While unit commitment (UC) is an off-line problem, economic dispatch (ED) is an area of online concern. The commitment decisions are made many weeks or months in advance. The decision to commit a generating unit to be able to produce electricity means that the power utility is willing to incur fixed costs related to unit startup in order to have that generating plant ready and available to produce electricity in real time. Generators with large start-up costs or long minimum-run times (such as large combustion turbines and nuclear power plants) cannot run optimally if their output is determined using a single-period analysis (a "period" in the electric power industry usually refers to a length of time of about an hour). Instead, their operation must be scheduled over a longer time horizon of days or even weeks. A power utility would need a forecast of demand weeks in advance before turning on a generator with a long minimum run time. They would need to look at the demand forecast over that period of weeks and decide the lowest-cost mix of generation plants that would meet the demand. This decision is referred to as the unit-commitment decision. While the process of dispatching generation plants to meet customer demands within a specified control footprint is known as "economic dispatch" or "optimal power flow."

Economic Load Dispatch are usually influence by factors such as high operating cost (fuel cost) and transmission losses. The Economic Load Dispatch requires the generation facilities to plan and forecast optimal energy dispatch. Hence the concept is the optimal selection of the generating units in such an economic manner that the total cost of supplying the dynamic requirements of the system is minimized[4].

\subsection{Formulation of ELD Problem}

The ELD problem is an optimization problem that determines the power output of each online generator that will result in a least cost system operating state.

$\begin{array}{ll}\text { Minimize } & f(x) \\ \text { Subject to } & g(x)\end{array}$

$h(x) \leq 0$

where $f(x)=$ objective function, $g(x)$ and $h(x)$ are set of equality and inequality constraints respectively.

The objective of the Economic Load Dispatch is to minimize the total operating cost of a power system by adjusting the power output of each of the generators connected to the grid, while satisfying the total load demand plus transmission losses within generator limits.

International Journal of Research Studies in Electrical and Electronics Engineering (IJRSEEE) Page | 21 
The generator cost curves are modeled with smooth quadratic function given by equation (2.4) subject to the equality and inequality constraints in equations (2.5) and (2.7) respectively[2].

Minimize the cost function,

$F_{i} P_{i}=\sum_{i=1}^{n_{g}}\left(\gamma_{i} P_{i}^{2}+\beta_{i} P_{i}+\alpha_{i}\right)$

Subject to power balance equation (equality constraint)

$\sum_{i=1}^{n_{g}} P_{i}=P_{D}+P_{L}$

The system losses can be determined by means of a power flow equation solution which is expressed in krons loss formula in equation (2.6).

$P_{L}=\sum_{i=1}^{n_{g}} \sum_{j=1}^{n_{g}} P_{i} B_{i j} P_{j}+\sum_{i=1}^{n_{g}} B_{0 i} P_{i}+B_{00}$

And the inequality constraints,

$P_{i(\min )} \leq P_{i} \leq P_{i(\max )}$

Where $F_{i} P_{i}$ is the total generation cost, $\alpha_{i}, \beta_{i}, \gamma_{i}$ are cost coefficients, $P_{D}$ is the load demand, $P_{i}$ is real power generation, $P_{L}$ is power transmission loss,$n_{g}$ is number of dispatchable generating plants, $P_{i(\min )}$ and $P_{i(\max )}$ are the minimum and maximum power generation limits respectively.also $B_{i j}, P_{j}, B_{0 i}$ and $B_{00}$ are B-coefficient[5].

\subsection{Operating Cost of a Thermal Plant}

The most efficient generator in the system does not guarantee minimum cost as it may be located in an area where fuel cost is high and also if power plant is located far from the load centre, transmission losses may be considerably higher and hence the power plant may become uneconomical.

The operating cost of a Thermal Plant plays an important role in the economic scheduling of different generating plants such that the total operating cost is minimal.

The input to the thermal power plant is generally measured in Btu/h or $\mathrm{Kcal} / \mathrm{h}$ and the output is measured in MW.

The input-output curve of a thermal unit is known as heat-rate curve. It is a graph drawn between fuel input in Btu/hr or $\mathrm{kcal} / \mathrm{hr}$ and power output in MW on the $\mathrm{x}$ - and y-axis respectively. A typical heatrate curve for a thermal unit takes the form as shown in Figure 2.1. Converting the ordinate of heatrate curve from Btu/h to N/h results in the fuel-cost curve as shown in Figure 2.2.

In all practical cases, the fuel cost of generator $i$ can be represented as a quadratic function of real power generation.

$C_{i}=\alpha_{i}+\beta_{i} P_{i}+\lambda_{i} P_{i}^{2}$

Incremental Fuel-cost curve is obtained by plotting the derivative of the fuel-cost curve versus the real power as shown in Figure 2.3. It is a measure of how costly it will be to produce the next increment of power. It is drawn by taking the incremental fuel cost in $\mathrm{N} / \mathrm{MWh}$ as an input on the $\mathrm{y}$-axis and real power in MW as output on the $\mathrm{x}$-axis.

$\frac{d C_{i}}{d P_{i}}=2 \gamma_{i} P_{i}+\beta_{i}$

The total operating cost includes the fuel cost, and the cost of labour, supplies and maintenance. These costs are assumed to be a fixed percentage of the fuel cost and are generally included in the incremental fuel-cost curve[6].

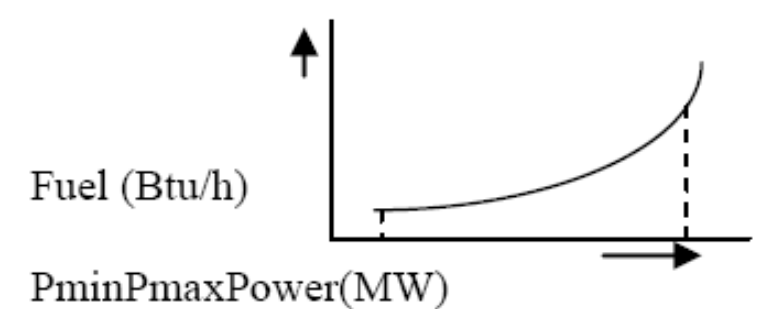

Figure2.1. Heat curve of a Thermal Unit. 


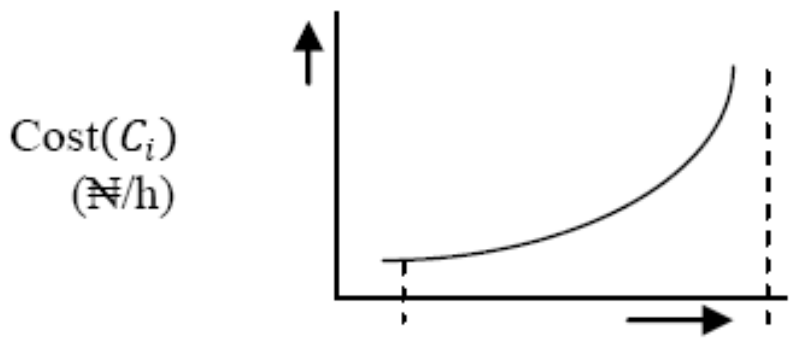

Figure2.2. Fuel-cost curve characteristic of a Thermal Unit

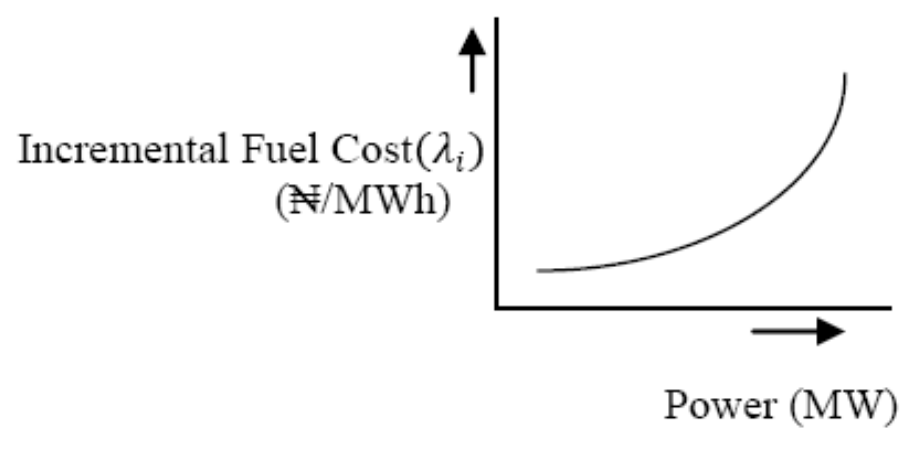

Figure2.3. Incremental Fuel-Cost Curve of a Thermal Unit

\section{Ant Colony Search Algorithm}

Ant Colony Search Algorithm (ACSA) is a population-based approach for solving combinatorial optimization problems that is inspired by the foraging behaviour of ants and their inherent ability to find the shortest path from a food source to their nest. ACSA is the result of research on computational intelligence approaches to combinatorial optimization originally conducted by Dr. Marco Dorigo, in collaboration with Alberto Colorni and Vittorio Maniezzo. The fundamental approach underlying ACSA is an iterative process in which a population of simple agents repeatedly construct candidate solutions; this construction process is probabilistically guided by heuristic information on the given problem instance as well as by a shared memory containing experience gathered by the ants in previous iteration. ACSA has been applied to a broad range of hard combinatorial problems. Among them, we have the classic Traveling Salesman Problem (TSP), where an individual must find the shortest route by which to visit a given number of destinations [7].

\subsection{Ant Colony Behaviour}

In many ants the visual system is very simple. Some species are completely blind. Communication between ants and their environment is often based on the use of chemical signals[13]. Pheromones are produced by ants and they deposit them on trials when walking in search of food. By sensing the pheromone, the following ants can find food. Inspirational source of ant colony algorithms is the double bridge experiment which depicts the ant behaviour. Ant nest is connected to food source via two paths of differing length. Initially, ants moverandomly and chose between shorter and longer path with equal probability. While walking, ants deposited pheromone. When choosing a path, ants chose with higher probability the path with the highest pheromone concentration. Ants choosing the short path will be first back with food. Therefore, trail on shorter path grows more quickly. Number of studies and surveys has been conducted by the researchers throughout the world for suggesting improved techniques to manage stress. Some of the reasons of work stress may be: Interpersonal causes, role demand, task demand, and structure of the organization[8].

\section{RESEARCH METHODOLOGY}

To solve the Economic Load Dispatch problem using Ant Colony Search Algorithm Technique, a MATLAB code was written. This MATLAB code was run on a Windows 7 Computer using Cost Coefficient Data collected from Egbin Thermal Power stations. This was carried out under variable load to determine their corresponding minimal generation fuel cost. 


\subsection{Ant Colony Search Algorithm For Economic Dispatch}

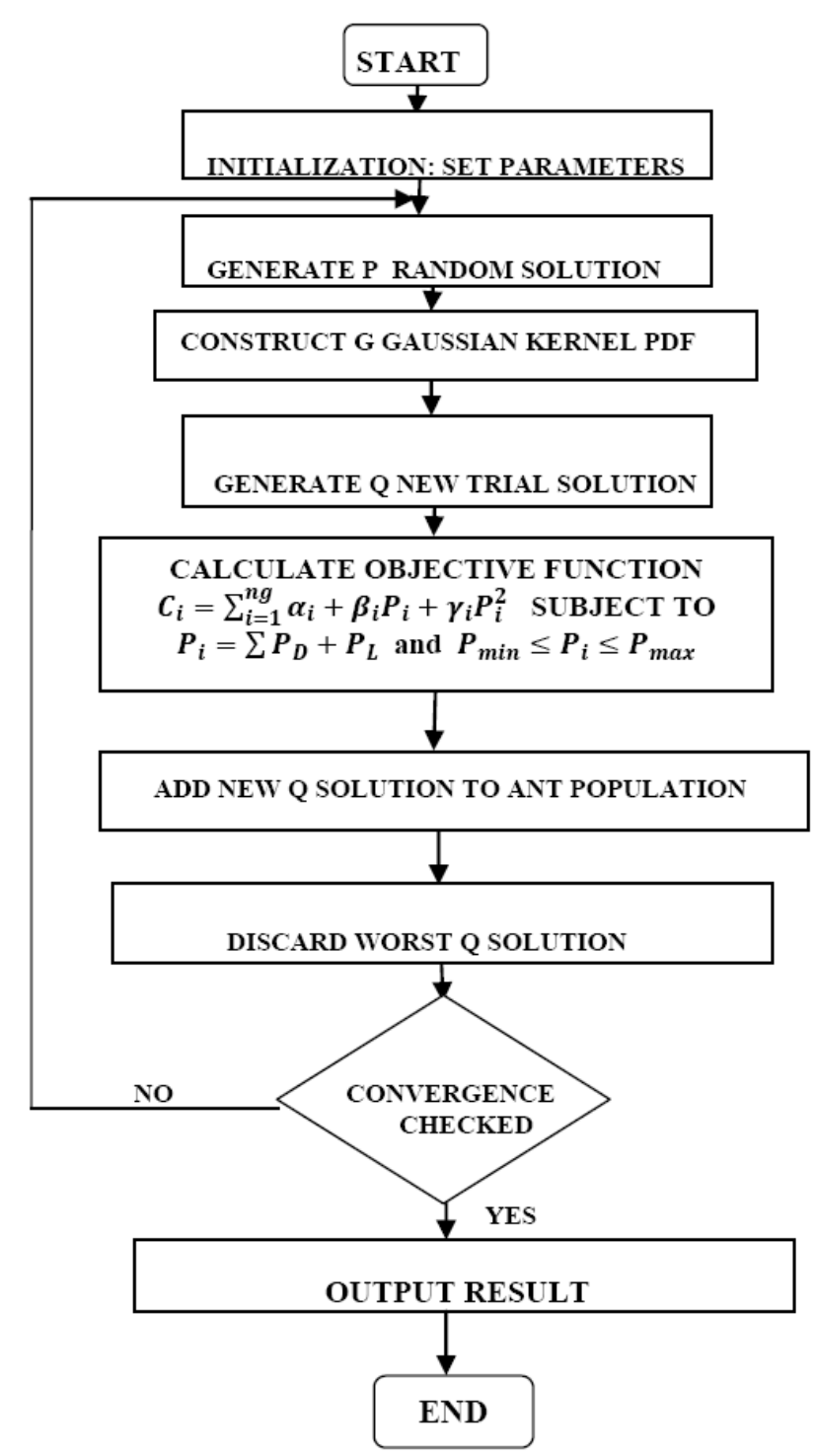

Figure4.1. Flow Chart for Ant Colony Search Algorithm for Economic Dispatch

\subsection{ACSA Algorithm Procedures}

\section{Step 1: Initialization}

In the beginning, the ACSA parameters have to be specified during initialization process.

During initialization, the following parameters need to be initialized.

MaxIt $=$ Maximum Number of Iterations.

$\mathrm{nAnt}=$ Number of Ants(Archives Population Size $)$.

nSample $=$ Number of New Ants(Sample Size $)$.

$\mathrm{q}=\quad$ Intensification Factor (Selection Pressure).

$\operatorname{zeta}(\zeta)=$ Deviation-Distance Ratio

\section{Step 2: Generate (P) Random Solution}

The first node will be selected by generating random number based on the uniform distribution, ranging from 1 to $n$. An ant constructs a solution for the decision variable

$x_{i}=1, \ldots \ldots . n$ by performing $\mathrm{n}$ construction steps. 


\section{Step 3: Construct G Gaussian Kernel PDF}

At each construction step, ant $\mathrm{k}$ applies a state transition rule in order to decide which node to be visited next. The ant $k$, which is currently positioned at current node $(r)$ will move to the next node $(s)$ by applying the state transition rule. At the $i^{\text {th }}$ construction step, the ant $\mathrm{k}$ chooses a value for the decision variable $x_{i}$ by sampling the Gaussian kernel PDF $G^{i}$ as described in equation 4.1

$G_{(x)}^{i}=\sum_{i=1}^{k} w_{i} g_{i}(x)(4.1)$

\section{Step 4: Generate Q new trial solution by sampling the G Gaussian Kernel Pdf}

$G^{i}$ can be defined by solving for the values of the vectors $\mu, \sigma a n d \omega$. The sampling process begins by first, choosing one of the Gaussian functions of the Gaussian kernel PDF. The probability $\rho_{l}$ of choosing the $l^{\text {th }}$ Gaussian function is

$\rho_{l}=\frac{\omega_{l}}{\sum_{r=1}^{k} \omega_{r}}(4.2)$

The process of sampling the chosen Gaussian function is the same as using a random number generator that is able to generate random numbers according to a normal distribution parameterized by the $l^{\text {th }}$ elements of the mean vector $\mu_{i}$ and standard deviation vector $\sigma^{i} l$. The whole process is performed for each dimension $i=1, \ldots \ldots . n$

\section{Step 5: Add new solution to population}

The Pheromone Update procedure is accomplished by performing an update on the solution archive $\mathrm{Q}$, the representation of pheromone information in ACSA. The set of newly generated solutions are added to the solution archive.

\section{Step 6: Discard worst Q Solution}

The number of worse ones are removed. This ensures that the best solutions guide the ants in the search process.

\section{Step 7: End Condition}

The algorithm stops the iteration when a maximum number of iterations (MaxIt) have been performed. If a better path is discovered in the process, it will be kept for the next reference. The best path selected between all iterations engages the optimal scheduling solution, the best solution found is updated and returned when the termination condition is met[9][10].

\section{Simulations AND Results}

The modeling and program development of ACSA was accomplished by using MATLAB R2008b software. All program have been run on Intel (R) $2.60 \mathrm{GHz}$ CPU, 2GB RAM, Window 7 Computer. To test the effectiveness and efficiency of this algorithm, a case study involving Egbin Thermal Power station was applied. The effect of ACSA on Economic Load Dispatch was investigated and evaluated. The system was evaluated using Lambda (Classical Approach) method against using ACSA.

Initial parameters to the proposed ACSA is presented as follows[11];

MaxIt $=200 ; \quad$ Maximum Number of Iterations

$\mathrm{nAnt}=10 ;$ Number of Ants(Archives Population Size)

nSample $=40 ; \quad$ Number of New Ants(Sample Size $)$

$\mathrm{q}=0.4 ; \quad$ Intensification Factor (Selection Pressure)

zeta $=0.88 ; \quad$ Deviation-Distance Ratio

\subsection{Case Study: Egbin Thermal Power Station}

This case study contains six $220 \mathrm{MW}$ generating units. The objective is to reduce the total operating cost, while conserving the system constraints under the allowable limits. While cutting down the operating cost, it was initially projected that the power loss might be reduced to minimal. This test case comprises six generating unit with quadratic functions as given in Table 5.1 
Evaluation of Economic Load Dispatch Problem in Power Generating Stations by the Use of Ant Colony Search Algorithms

Table5.1. Cost coefficients for the six generators at Egbin Power Station

\begin{tabular}{|c|c|c|c|}
\hline $\begin{array}{c}\text { Gen } \\
\text { units }\end{array}$ & $\begin{array}{c}(\boldsymbol{\alpha}) \\
\text { alpha(N/hr) }\end{array}$ & $\begin{array}{c}(\boldsymbol{\beta}) \\
\text { beta(N/MWhr) }\end{array}$ & $\begin{array}{c}(\boldsymbol{\gamma}) \\
\text { gamma(N/MW } \mathbf{~ h r ) ~}\end{array}$ \\
\hline 1 & 2131.1667 & 13.1 & 0.186 \\
\hline 2 & 2131.1667 & 13.1 & 0.186 \\
\hline 3 & 2131.1667 & 13.1 & 0.186 \\
\hline 4 & 2131.1667 & 13.1 & 0.186 \\
\hline 5 & 2131.1667 & 13.1 & 0.186 \\
\hline 6 & 2131.1667 & 13.1 & 0.186 \\
\hline
\end{tabular}

Source: [12]

Table5.2. Generating limits for 6 generators at Egbin Power Station

\begin{tabular}{|c|c|c|}
\hline Generating Units & Minimum(MW) & Maximum(MW) \\
\hline 1 & 55 & 220 \\
\hline 2 & 55 & 220 \\
\hline 3 & 55 & 220 \\
\hline 4 & 55 & 220 \\
\hline 5 & 55 & 220 \\
\hline 6 & 55 & 220 \\
\hline
\end{tabular}

Source:[12]

Table5.3. Power Losses (B- coefficient) of Nigerian Power System

\begin{tabular}{|lllllll|}
\hline \multicolumn{1}{|c|}{ B-coefficient data } \\
\hline 0.0009919 & 0.0001556 & -0.0001651 & -0.0001736 & -0.0001631 & 0.0001481 \\
0.0001417 & 0.0001209 & -0.0001278 & -0.0001344 & -0.0001263 & 0.0001155 \\
-0.0001324 & -0.0001169 & 0.0001256 & 0.0001321 & 0.0001238 & -0.0001102 \\
-0.0001408 & -0.0001238 & -0.0001328 & 0.0001396 & 0.0001309 & -0.0001169 \\
-0.0001332 & -0.0001170 & 0.0001254 & 0.0001319 & 0.0001237 & -0.0001105 \\
0.0001481 & -0.0001235 & -0.0001290 & -0.0001356 & -0.0001276 & 0.0001190 \\
\hline
\end{tabular}

Source: [12]

Table5.4. Results obtained from Egbin Power Station (Comparison between Lambda Method and ACSA).

Power Demand =500MW, Lambda $=43.38$

\begin{tabular}{|c|c|c|}
\hline Outputs & Lambda Method & ACSA \\
\hline G1 & 71.116 & 70.401 \\
\hline G2 & 84.635 & 86.358 \\
\hline G3 & 83.273 & 87.101 \\
\hline G4 & 88.531 & 86.772 \\
\hline G5 & 83.304 & 83.535 \\
\hline G6 & 89.462 & 85.957 \\
\hline Total Generated(MW) & 500.320 & 500.124 \\
\hline Total Losses(MW) & 0.320 & 0.124 \\
\hline Total Generation Cost (N/hr) & 27141.209 & 27131.389 \\
\hline
\end{tabular}

Table5.5. Results obtained from Egbin Power Station (Comparison between Lambda Method and ACSA).

Power Demand $=600 \mathrm{MW}$, Lambda $=49.64$

\begin{tabular}{|c|c|c|}
\hline Outputs & Lambda Method & ACSA \\
\hline G1 & 83.605 & 84.810 \\
\hline G2 & 101.721 & 102.410 \\
\hline G3 & 99.768 & 101.220 \\
\hline G4 & 106.953 & 104.442 \\
\hline G5 & 99.810 & 101.784 \\
\hline G6 & 108.345 & 105.372 \\
\hline Total Generated Power(MW) & 600.201 & 600.040 \\
\hline Total Loss(MW) & 0.201 & 0.040 \\
\hline Total Generation Cost( $/ \mathrm{hr})$ & 31889.629 & 31862.792 \\
\hline
\end{tabular}


Table5.6. Results obtained from Egbin Power Station (Comparison between Lambda Method and ACSA).

Power Demand =700MW, Lambda $=\mathbf{5 7 . 0 0}$

\begin{tabular}{|c|c|c|}
\hline Outputs & Lambda Method & ACSA \\
\hline G1 & 95.629 & 95.722 \\
\hline G2 & 118.895 & 124.212 \\
\hline G3 & 116.221 & 120.247 \\
\hline G4 & 125.627 & 120.903 \\
\hline G5 & 116.280 & 116.445 \\
\hline G6 & 127.593 & 122.477 \\
\hline Total Generated Power(MW) & 700.246 & 700.006 \\
\hline Total Loss(MW) & 0.246 & 0.006 \\
\hline Total Generation Cost(A/hr) & 37281.328 & 37251.525 \\
\hline
\end{tabular}

\subsection{Discussion of Results}

Results from Tables 5.4, 5.5, 5.6, 5.7, 5.8 and 5.9 for the case study, shows that ACSA has effectively minimized the cost of generation as compared to the Lambda method. Hence the ACSA has offered better results when compared with the Lambda method. The total generation costs and transmission losses obtained via ACSA are also lower when compared with the values obtained using the Lambda method (Classical Approach).Also Figures 5.1,5.2,5.3 and 5.4 shows the convergence nature of ACSA..

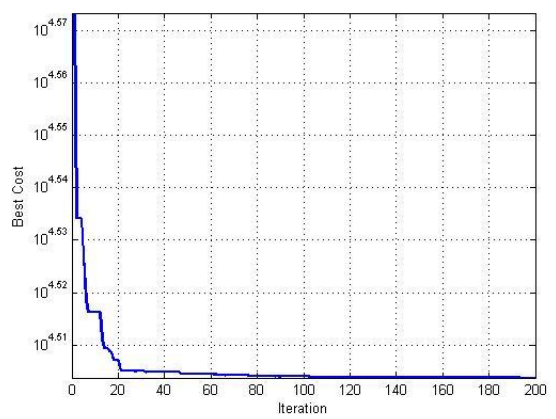

Figure5.1. Cost curve of ACSA using Power demand of 500MW

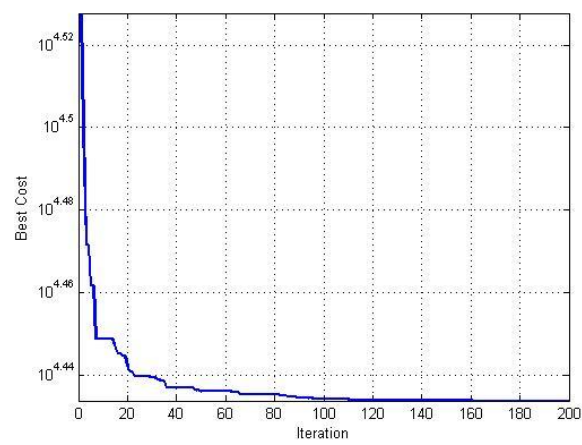

Figure5.2. Cost curve of ACSA using Power demand of 600MW

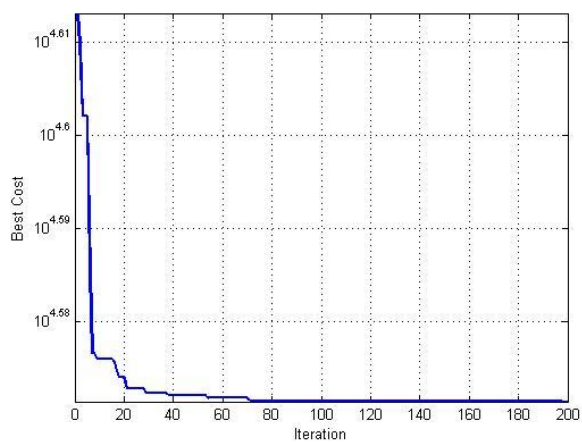

Figure5.3. Cost curve of ACSA using Power demand of 700MW 


\section{CONCLUSION}

The developed ACSA technique has been successfully applied for the solution of the economic load dispatch in power system in this paper. The successful implementation of the proposed ACSA on Egbin thermal station considering transmission losses proved to be the required method for solving economic load dispatch of real power generation problem. It has been observed that the ACSA technique is capable of optimizing any given ELD problem under variable load demand. From the analysis of the proposed ACSA technique which was implemented in MATLAB environment using Egbin Thermal Power Station as case study considering transmission losses, proves that ACSA is highly efficient, accurate and has capacity to minimize the fuel cost of generators and satisfies each and every constraint. Thus, ACSA technique can be successfully applied to solve ELD problems in the real world power systems.

\section{REFERENCES}

[1] Thakral. D.R and Rai H.M (2008) Ant Colony Optimization: A new approach for economic load dispatch.IJREAS, vol.2,Is.11, ISSN:2249-3905.

[2] A. Y. Abdelaziz, S. F. Mekhamer, M. A. L. Badr and M. Z. Kamh, (2008). Economic Dispatch Using an Enhanced Hopfield Neural Network', Electric Power Components and Systems Journal, Vol. 36, No. 7 pp. 719-732.

[3] Aristidis Vlachos, Isidoros Petikas \& Simos Kyriakides. (2011). A Continuous Ant Colony (CANT) algorithm solving the Economic Load Dispatch (ELD) Problem, Journal of Information and Optimization Sciences, 32:1, 1-13, DOI: 10.1080/02522667.2011.10700039

[4] Bonabeau E, Dorigo M, Theraulaz G.(1999). Swarm Intelligence: From Natural to Artificial Systems. Journal of Artificial Societies and Social Simulation, Pp.320.

[5] Ioannis Kara konstantis \& Aristidis Vlachos (2015). Ant Colony Optimization for Continuous Domains applied to Emission and Economic Dispatch Problems, Journal of Information and Optimization Sciences, 36:1-2, 23-42 DOI: 10.1080/02522667.2014.932094.

[6] Lin,J, Chen.C.L, Tsai.S.F \&Yuan.C.(2015). New intelligent particle swarm optimization algorithm for solving economic dispatch with valve-point effects. Journal of Marine Science and Technology, 23(1), 44-53.

[7] Swarup K.S (2005).Ant colony optimization for economic generator scheduling and load dispatch. Proceedings of the $6^{\text {th }}$ WSEAS int. Conf. on evolutionary computing ,Lisbon.Pp.167175.

[8] M. Dorigo\& T. Stutzle. (2004): Ant Colony Optimization. MIT Press/Bradford Books, Page(s) $16-40$.

[9] Ioannis Karakonstantis \& Aristidis Vlachos (2015). Ant Colony Optimization for Continuous Domains applied to Emission and Economic Dispatch Problems, Journal of Information and Optimization Sciences, 36:1-2, 23-42 DOI: 10.1080/02522667.2014.932094.

[10] Socha. K and M. Dorigo (2006). Ant colony optimization for continuous domains. European Journal of Operational Research,

[11] Mostapha Kalami Heris.S, (2015). Ant Colony Optimization for Solving Economic Load Dispatch (MATLAB programme) .Publisher: Yarpizwww.yarpiz.comretrieved on 12/07/2017.

[12] Onah C. O, Agber J. U and Ikule F. T (2016). Optimal Dispatch of Real Power Generation Using Particle Swarm Optimization: A Case Study Of Egbin Thermal Station. Iinternatiomal Journal of Modern Trend in Engineering and Research. Volume 3, Issue 12 


\section{AUTHORS' BIOGRAPHY}

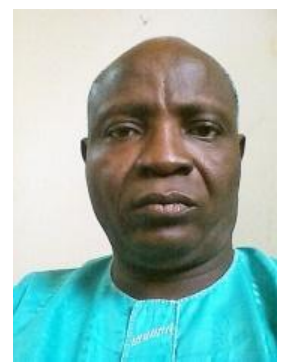

Engr. Dr. M. N. Nwohu, is an Associate Professor in Electrical Power Engineering. He received his bachelor of Engineering (B.Eng.) in Power Systems Engineering Technology from Federal University of Technology, Owerri, Nigeria, in 1988. He got his Masters degree in Power and Machines from University of Benin, Nigeria in 1994. In 2007, he badged on his Doctor of Philosophy (PhD) Degree in Power Systems Engineering from Abubakar Tafawa Balewa University, Bauchi, Nigeria. His research interest includes Power System Analysis, Power System Stability and Control, Application of FACTS devices to Power Systems, System Simulation, Power Quality and Numerical Analysis. He was Head of Department of Electrical and Computer Engineering, Federal University of Technology, Minna in 2002/2003 session and Head of Department of Computer Engineering of same University in 2011/2012 session. He has authored over forty(40) refereed publications in International journals and Conference proceedings. An author of a textbook captioned Voltage Stability Enhancement of the Nigerian Grid System using FACTS. He is COREN registered and a member of IAENG, IET, NSE and ISDS.

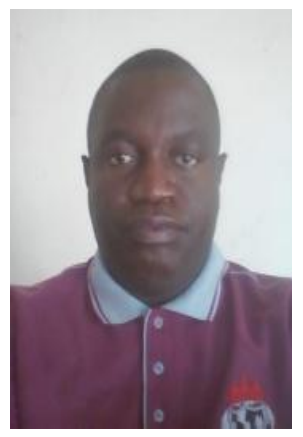

Mr. Osaremwinda Osarobo Paul, was born on 10 July 1973 in Benin City. He attended Baptist High School in Benin City where he obtained his SSCE in 1991. In 1997, he obtained his Bachelor degree in Electrical/Electronics Engineering. His research interest is in Power and Telecommunications and has been a contractor to both Power and Telecommunications firms. He holds a CCNP in networking. Currently, he is a Postgraduate student in Electrical Power and Machines, Federal University of Technology, Minna 